\title{
A new management approach based on Additive Manufacturing technologies and Industry 4.0 requirements
}

\author{
Patalas-Maliszewska, J. ${ }^{\mathrm{a},{ }^{*}}$, Topczak, M. ${ }^{\mathrm{a}}$ \\ ${ }^{a}$ Institute of Mechanical Engineering, University of Zielona Góra, Poland
}

\begin{abstract}
A B S T R A C T
Nowadays, it is necessary to formulate and implement a development strategy in manufacturing enterprises, in line with the assumptions of the Industry 4.0 concept. In this context, a gap in the research has been observed in effective management methods, in order to gain a competitive advantage through the implementation and use of Additive Manufacturing (AM) technologies. The main purpose of the study is to build a new approach to management, based on the implementation of new AM technologies and good practice. This paper uses the detailed literature studies and results from the empirical research of some 250 Polish manufacturing enterprises; this material contains a sample thereof, processed into a new approach. The major contributions of the work are as follows: (1) identification of current management areas in which manufacturing companies focus their activities, in the context of Industry $4.0,(2)$ the establishment of the correlation between gaining a competitive advantage and implementing AM technologies in the context of Industry 4.0, (3) Defining the so-called AM4.0CARD as a new management approach, based on AM technologies and the requirements of Industry 4.0. Managers of manufacturing enterprises, thanks to the use of the proposed approach, may take a strategic decision, regarding the implementation of AM technologies, due to the possibility of forecasting the impact of such an investment on the improvement of the company's competitive advantage.
\end{abstract}

\author{
ARTICLE INFO \\ Keywords: \\ Smart manufacturing; \\ Industry 4.0; \\ Additive Manufacturing (AM); \\ 3D printing; \\ Strategy; \\ Management; \\ Empirical research \\ Competitive advantage; \\ Balanced scorecard \\ *Corresponding author: \\ j.patalas-maliszewska@iim.uz.zgora.pl \\ (Patalas-Maliszewska, J.) \\ Article history: \\ Received 14 June 2020 \\ Revised 4 March 2021 \\ Accepted 6 March 2021

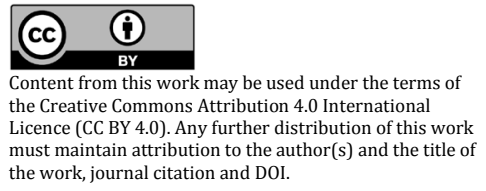

\section{References}

[1] Sarı, T., Güleș, H.K., Yiğitol, B. (2020). Awareness and readiness of Industry 4.0: The case of Turkish manufacturing industry, Advances in Production Engineering \& Management, Vol. 15, No. 1, 57-68, doi: 10.14743/ apem2020.1.349.

[2] Madhikermi, M., Kubler, S., Robert, J., Buda, A., Främling, K. (2016). Data quality assessment of maintenance reporting procedures, Expert Systems with Applications, Vol. 63, 145-164, doi: 10.1016/i.eswa.2016.06.043.

[3] Medic, N., Anisic, Z., Lalic, B., Marjanovic, U., Brezocnik, M. (2019). Hybrid fuzzy multi-attribute decision making model for evaluation of advanced digital technologies in manufacturing: Industry 4.0 perspective, Advances in Production Engineering \& Management, Vol. 14, No. 4, 483-493, doi: 10.14743/apem2019.4.343.

[4] Horvat, D., Stahlecker, T., Zenker, A., Lerch, C., Mladineo, M. (2018). A conceptual approach to analysing manufacturing companies' profiles concerning Industry 4.0 in emerging economies, Procedia Manufacturing, Vol. 17, 419-426, doi: 10.1016/i.promfg.2018.10.065.

[5] Hozdić, E., Butala, P. (2020). Concept of socio-cyber-physical work systems for Industry 4.0, Tehnički Vjesnik Technical Gazette, Vol. 27, No. 2, 399-410, doi: 10.17559/TV-20170803142215.

[6] Patalas-Maliszewska, J.,Topczak, M., Kłos, S. (2020). The level of the additive manufacturing technology use in Polish metal and automotive manufacturing enterprises, Applied Sciences, Vol. 10, No. 3, Article No. 735, doi: 10.3390/app10030735. 
[7] Mandic, M., Galeta, T., Raos, P., Jugovic, V. (2016). Dimensional accuracy of camera casing models 3D printed on Mcor IRIS: A case study, Advances in Production Engineering \& Management, Vol. 11, No. 4, 324-332, doi: 10.14743/apem2016.4.230.

[8] Haleem, A., Javaid, M. (2019). Additive manufacturing applications in Industry 4.0: A review, Journal of Industrial Integration and Management, Vol. 4, No. 4, Article No. 1930001, doi: 10.1142/S2424862219300011.

[9] Bobko, P. (2001). Correlation and regression: Applications for industrial organizational psychology and management, Second Edition, Sage Publications, Thousand Oaks, California, USA, doi: 10.4135/9781412983815.

[10] Rabbani, A., Zamani, M., Yazdani-Chamzini, A., Zavadskas, E.K. (2016). Proposing a new integrated model based on sustainability balanced scorecard (SBSC) and MCDM approaches by using linguistic variables for the performance evaluation of oil producing companies, Expert Systems with Applications, Vol. 41, No. 16, 7316-7327, doi: 10.1016/i.eswa.2014.05.023.

[11] Antosz, K., Pasko, L., Gola, A. (2019). The use of intelligent systems to support the decision-making process in lean maintenance management, IFAC PapersOnLine, Vol. 52, No. 10, 148-153, doi: 10.1016/j.ifacol.2019.10.037.

[12] Wagner, S., Goossen, M.C. (2018). Knowing me, knowing you: Inventor mobility and the formation of technologyoriented alliances, Academy of Management Journal, Vol. 61, No. 6, 2026-2052, doi: 10.5465/amj.2016.0818.

[13] Hengst, I.-A., Jarzabkowski, P., Hoegl, M., Muethel, M. (2020). Toward a process theory of making sustainability strategies legitimate in action, Academy of Management Journal, Vol. 63, No. 1, 246-271, doi: 10.5465/amj.2016. 0960.

[14] Patalas-Maliszewska, J., Kłos, S. (2019). The methodology of the S-ERP system employment for small and medium manufacturing companies, IFAC-PapersOnLine, Vol. 52, No. 10, 85-90, doi: 10.1016/j.ifacol.2019.10.004.

[15] Uzunca, B. (2018). A competence-based view of industry evolution: The impact of submarket convergence on incumbent-entrant dynamics, Academy of Management Journal, Vol. 61, No. 2, 738-768, doi: 10.5465/amj.2015. 1080.

[16] Kilduff, G.J. (2019). Interfirm relational rivalry: Implications for competitive strategy, Academy of Management Review, Vol. 44, No. 4, 775-799, doi: 10.5465/amr.2017.0257.

[17] Medeiros, J., Vasconcelos, A., Silva, C., Goulão, M. (2018). Quality of software requirements specification in agile projects: A cross-case analysis of six companies, Journal of Systems and Software, Vol. 142, 171-194, doi: 10.1016/i.jss.2018.04.064.

[18] Ricciardi, F., Zardini, A., Rossignoli, C. (2018). Organizational integration of the IT function: A key enabler of firm capabilities and performance, Journal of Innovation \& Knowledge, Vol. 3, No. 3, 93-107, doi: 10.1016/i.jik.2017. $\underline{02.003}$

[19] Campos, C., Grangel, G. (2018). A domain-specific modelling language for corporate social responsibility (CSR), Computers in Industry, Vol. 97, 97-110, doi: 10.1016/i.compind.2018.01.007.

[20] Chen, T.-Y. (2018). A novel risk evaluation method of technological innovation using an inferior ratio-based assignment model in the face of complex uncertainty, Expert Systems with Applications, Vol. 95, 333-350, doi: 10.1016/i.eswa.2017.11.038.

[21] Jafari Sadeghi, V., Nkongolo-Bakenda, J.-M., Anderson, R.B., Dana, L.-P. (2019). An institution-based view of international entrepreneurship: A comparison of context-based and universal determinants in developing and economically advanced countries, International Business Review, Vol. 28, No. 6, Article No. 101588, doi: 10.1016/ j.ibusrev.2019.101588.

[22] Sun, W., Price, J., Ding, Y. (2019). The longitudinal effects of internationalization on firm performance: The moderating role of marketing capability, Journal of Business Research, Vol. 95, 326-337, doi: 10.1016/i.jbusres. 2018.08.014.

[23] Zou, W., Brax, S.A., Rajala, R. (2018). Complexity in product-service systems: Review and framework, Procedia CIRP, Vol. 73, 3-8, doi: 10.1016/i.procir.2018.03.319.

[24] Shihundla, T.B., Mpofu, K., Adenuga, O.T. (2019). Integrating product-service systems into the manufacturing industry: Industry 4.0 perspectives, Procedia CIRP, Vol. 83, 8-13, doi: 10.1016/j.procir.2019.03.147.

[25] Xu, Y., Koivumäki, T. (2019). Digital business model effectuation: An agile approach, Computers in Human Behavior, Vol. 95, 307-314, doi: 10.1016/j.chb.2018.10.021.

[26] Kang, T., Baek, C., Lee, J.-D. (2019). Effects of knowledge accumulation strategies through experience and experimentation on firm growth, Technological Forecasting and Social Change, Vol. 144, 169-181, doi: 10.1016/ j.techfore.2019.04.003.

[27] Mahdi, O.R., Nassar, I.A., Almsafir, M.K. (2019). Knowledge management processes and sustainable competitive advantage: An empirical examination in private universities, Journal of Business Research, Vol. 94, 320-334, doi: 10.1016/j.jbusres.2018.02.013.

[28] Salimi, N., Rezaei, J. (2018). Evaluating firms' R\&D performance using best worst method, Evaluation and Program Planning, Vol. 66, 147-155, doi: 10.1016/i.evalprogplan.2017.10.002.

[29] Abdel-Baset, M., Chang, V., Gamal, A. (2019). Evaluation of the green supply chain management practices: A novel neutrosophic approach, Computers in Industry, Vol. 108, 210-220, doi: 10.1016/i.compind.2019.02.013.

[30] Ren, M., Ren, L., Jain, H. (2018). Manufacturing service composition model based on synergy effect: A social network analysis approach, Applied Soft Computing, Vol. 70, 288-300, doi: 10.1016/i.asoc.2018.05.039.

[31] Riesener, M., Dölle, C., Schuh, G., Lauf, H., Jank, M.-H. (2019). Performance-driven and company goal-orientated design of product portfolios: A methodological framework, Procedia CIRP, Vol. 84, 725-730, doi: 10.1016/ j.procir.2019.03.267.

[32] Schuh, G., Doelle, C., Koch, J., Grutza, P. (2018). Identification of the cost-benefit-optimal product configuration, Procedia CIRP, Vol. 70, 386-391, doi: 10.1016/i.procir.2018.03.294.

[33] Hamzeh, R., Xu, X. (2019). Technology selection methods and applications in manufacturing: A review from 1990 to 2017, Computers \& Industrial Engineering, Vol. 138, Article No. 106123, doi: 10.1016/i.cie.2019.106123. 
[34] Ghadimi, P., Ghassemi Toosi, F., Heavey, C. (2018). A multi-agent systems approach for sustainable supplier selection and order allocation in a partnership supply chain, European Journal of Operational Research, Vol. 269, No. 1, 286-301, doi: 10.1016/j.ejor.2017.07.014.

[35] Omar, Y.M., Minoufekr, M., Plapper, P. (2019). Business analytics in manufacturing: Current trends, challenges and pathway to market leadership, Operations Research Perspectives, Vol. 6, Article No. 100127, doi: 10.1016/ j.orp.2019.100127.

[36] Mittal, S., Khan, M.A., Romero, D., Wuest, T. (2019). Building blocks for adopting smart manufacturing, Procedia Manufacturing, Vol. 34, 978-985, doi: 10.1016/i.promfg.2019.06.098.

[37] Enke, J., Metternich, J., Bentz, D., Klaes, P.-J. (2018). Systematic learning factory improvement based on maturity level assessment, Procedia Manufacturing, Vol. 23, 51-56, doi: 10.1016/i.promfg.2018.03.160.

[38] Sousa, P., Tereso, A., Alves, A., Gomes, L. (2018). Implementation of project management and lean production practices in a SME Portuguese innovation company, Procedia Computer Science, Vol. 138, 867-874, doi: 10.1016/ j.procs.2018.10.113.

[39] Riesener, M., Rebentisch, E., Doelle, C., Kuhn, M., Brockmann, S. (2019). Methodology for the design of agile product development networks, Procedia CIRP, Vol. 84, 1029-1034, doi: 10.1016/i.procir.2019.04.172.

[40] Aschehoug, S.H., Lodgaard, E., Schulte, K.Ø. (2019). Success factors for open innovation in Norwegian manufacturing, Procedia CIRP, Vol. 84, 1107-1111, doi: 10.1016/i.procir.2019.04.279. 
APEM
Advances in Production Engineering \& Management Letnik 16 | Številka 1 | Marec 2021 | Strani 125-135
ISSN 1854-6250

Spletna stran: apem-journal.org

Izvirni znanstveni članek

\title{
Nov pristop upravljanja, ki temelji na dodajalni tehnologiji in zahtevah industrije $\mathbf{4 . 0}$
}

\author{
Patalas-Maliszewska, J. ${ }^{\mathrm{a},}{ }^{*}$, Topczak, $\mathrm{M} .^{\mathrm{a}}$ \\ anstitute of Mechanical Engineering, University of Zielona Góra, Poland
}

\section{POVZETEK}

$\mathrm{V}$ današnjem času je pomembno oblikovati in izvajati strategijo razvoja proizvodnih podjetij, skladno s predpostavkami koncepta Industrije 4.0. V tem kontekstu je bila opažena vrzel v raziskavah o učinkovitih metodah upravljanja, s katerimi bi pridobili konkurenčno prednost, in ki temeljijo na uporabi dodajalne tehnologije (AM). Glavni namen študije je zgraditi nov pristop $\mathrm{k}$ vodenju, ki temelji na vpeljavi novih AM tehnologij in dobrih praks. Ta članek uporablja izsledke podrobne študije literature in rezultate empirične raziskave približno 250 poljskih proizvodnih podjetij. Glavni prispevki raziskave so: 1.) identifikacija trenutnih področij vodenja, na katera proizvodna podjetja usmerjajo svoje aktivnosti, v kontekstu industrije 4.0, 2.) vzpostavitev korelacije med pridobivanjem konkurenčne prednosti in vpeljavo AM tehnologije $\mathrm{v}$ kontekstu industrije 4.0, 3.) opredelitev t. i. AM4.0CARD kot novega pristopa upravljanja, ki temelji na AM tehnologijah in zahtevah industrije 4.0. Vodje proizvodnih podjetij lahko s pomočjo uporabe predlaganega pristopa sprejmejo strateško odločitev o uvedbi AM tehnologij, saj jim pristop omogoča napoved vpliva takšne investicije na izboljšanje konkurenčne prednosti podjetja.

\section{PODATKI O ČLANKU}

Ključne besede:

Pametna proizvodnja;

Industrija 4.0;

Dodajalna tehnologija (AM);

3D tiskanje;

Strategija;

Upravljanje;

Empirične raziskave;

Konkurenčna prednost;

Uravnotežen kazalnik

* Kontaktna oseba:

j.patalas-maliszewska@iim.uz.zgora.pl

(Patalas-Maliszewska, J.)

Zgodovina članka:

Prejet 14. junija 2020

Popravljen 4. marca 2021

Sprejet 6. marca 2021

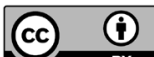

Content from this work may be used under the terms of the Creative Commons Attribution 4.0 International Licence (CC BY 4.0). Any further distribution of this work Licence (CC BY 4.0). Any further distribution of this work
must maintain attribution to the author(s) and the title of the work, journal citation and DOI. 